\title{
Comparative Experimental Studies of Angiotensin-Converting Enzyme Inhibitors and Angiotensin II Receptor Blockers against Two Different Models of Angiogenesis
}

\author{
Vijay Rajeshwarrao Chidrawar ${ }^{1, *}$, Samreen Soomro ${ }^{2}$ \\ 'Department of Pharmacology and Toxicology, Faculty of Pharmacy, Northern Border University, Kingdom of SAUDI ARABIA. \\ 2Department of Basic Health Sciences, Faculty of Pharmacy, Northern Border University, Kingdom of SAUDI ARABIA.
}

\begin{abstract}
Objective: Angiogenesis, the formation of new capillary blood vessels. We have compared the effect of two Angiotensin-Converting Enzyme inhibitors (ACEIs) and two Angiotensin Receptor Blockers (ARBs) for its anti-angiogenic property to pinpoint their role in capillary growth. Methods: The antiangiogenic activity of ACEls (Lisinopril, Ramipril) and ARBs (Losartan, Valsartan) were evaluated by in-ovo using and in-vivo methods by using chick embryo Chorioallantoic membrane (CAM) assay and sponge implantation method respectively. All the test drugs were tested at three dose level and Suramin was considered as standard. Before coming to the final conclusion various parameters were studied like angiogenic score, the number of branching points and micro-vessels in CAM assay, whereas the determination of $\mathrm{Hb}$ content, wet weight of the implants and VEGF was carried out in sponge implantation model. Results: Among all the drugs Losartan, AT1R blocker has shown a promising anti-angiogenic effect against both the models. Valsartan has also shown modest anti-angiogenic activity but not as good as Losartan. Losartan has shown significant $(p<0.005)$ dose-
\end{abstract}

dependent decrease in the number of blood vessels, new branching points, angiogenic score and the decrease in wet weight, $\mathrm{Hb}$ content and VEGF content in the implants compared to normal control group. There was no significant difference were observed in any of the parameters by ACEI. Conclusion: Losartan possesses a significant potential to inhibit angiogenesis and this property could be useful in controlling metastasis in malignant cancerous tumors.

Key words: Angiogenesis, CAM Assay, Sponge implantation, ACEls, ARBs.

Correspondence

Dr. Vijay Rajeshwarrao Chidrawar, Assistant Professor, Department of Pharmacology and Toxicology, Faculty of Pharmacy, Northern Border University, Kingdom of SAUDI ARABIA.

Phone: +966-580665011

Email: vijay_pharmacology@yhaoo.com

DOI: 10.5530/jyp.2019.11.35

\section{INTRODUCTION}

Angiogenesis is the formation of new blood vessels from the preexisting one. It plays an important role in many physiological and pathological conditions. ${ }^{1}$ Over angiogenesis may be a reason for tumor survival and metastasis whereas vessel growth could benefit in case of baldness, neurodegenerative ills and heart attack, also helpful to bypass the clots in blood vessels (Occlusion) and also in tissue repair. ${ }^{2}$ Angiotensin-Converting Enzyme inhibitors (ACEIs) and Angiotensin Receptor Blockers (ARBs) are the two major categories of the drugs which are used widely for many cardiovascular complications acting by modulating the RAAS (Renin-angiotensin-aldosterone-system) system. Earlier literature suggests that ACEIs might have anti-tumor properties. ${ }^{3,4}$ Angiotensin II is a major culprit for many diseases including tumor growth promoter via angiogenesis from activation of the Vascular endothelial growth factor (VEGF) pathway. ${ }^{5-7}$ Epidemiological data have some controversial findings of the use of ACEIs was linked with reduced risk of developing cancers including solid tumors. ${ }^{8-11}$

ARBs are successful primarily in the therapy of hypertension, but may also be beneficial in patients with intolerance to ACEIs for the treatment of several cardiovascular diseases, such as stable coronary heart disease, the state after acute myocardial infarction and heart failure. ${ }^{12}$ However, experimental studies in the recent decade have shown yet unmapped areas of the RAAS with certain effects and clinical consequences, which cannot be disregarded in the use of ARBs.

ARBs exert their main clinical effects by inhibiting AT1R and they have an inhibitory effect on tumor growth. However, earlier data reveals that overuse of ARBs (Angiotensin 1 receptor blockers) can induce cancer via overstimulation of angiotensin II receptors. ${ }^{12}$

Based upon the above observations present study was undertaken to sort out the exact role of ACEI and ARBs on neovascularization by using various models of angiogenesis. In the present study, we have randomly selected two ACEI i.e. Ramipril and Lisinopril and two ARBs i.e. losartan and valsartan and screened by in-ovo and in-vivo methods by selecting 3 doses of each test drug and considering various parameters involved in neovascularization.

\section{MATERIAL AND METHODS}

\section{Chemicals and Instruments}

Ramipril, Lisinopril, Losartan Potassium and Valsartan were purchased from Santa Cruz, USA, supplied by Bio Medical Sciences Est. Saudi Arabia. Dulbecco's modified Eagle's medium. All the chemicals used in the research are of AR grade. Fully automatic egg incubator with temperature humidity controller facility, Kwality micro, model 21; digital Sight series digital camera Nikon-DS-Fi3, polyester polyurethane sponge etc.

\section{Animal}

Before commencing the experiments National Committee of Bio Ethics (NCEB) approval was taken. Guidelines said by the NCEB, Saudi Arabia was strictly followed. All the studies were sanctioned by the Local committee of Bio Ethics (LCBE), (Ref: 169/39/19/D). Wistar male albino mice weighing in between 25-30 g were procured from the cen- 
tral animal house (Animal Biosafety Level 1) at Northern Border University, Saudi Arabia. The animals were maintained at a controlled temperature $\left(22-25^{\circ} \mathrm{C}, 45 \%\right.$ humidity) on a $12: 12$-h dark-light cycle. The animals were facilitated with satisfactory standard diet and eco-friendly conditions throughout the experiment. All the experiments were carried out between 9:00-16:00 hrs.

\section{Chick chorioallantoic membrane (CAM) assay}

CAM assay is considered as the 'Gold model' for the screening of drugs for angiogenic activity. In the present study, the CAM model of angiogenesis was carried out as per the procedure described in the earlier literature with some modification. ${ }^{13}$ Briefly, fertilized eggs of white leghorn hen collected at day ' 0 ' from the local hatchery and incubated at $37^{\circ} \mathrm{C}$ for the next $48 \mathrm{hrs}$ by keeping them vertically. After washing all the eggs with the distilled water, eggs were again incubated at $37^{\circ} \mathrm{C}$ for the next three days. Use of soap water and alcohol was avoided because in our pilot study we observed $70-80 \%$ mortality in the eggs. During these three days of incubation period eggs were rotated at $180^{\circ} \mathrm{C}$ for every $6 \mathrm{hrs}$. Then after, $2-3 \mathrm{ml}$ of the albumin was taken out by drilling a small hole at the narrow end of the egg, this help to detach the developing CAM from the inner eggshell. Immediately, hole was sealed with the cellophane tape and eggs were incubated as before. On the $7^{\text {th }}$ day of the study, a small square window $(3 \mathrm{~mm} \times 3 \mathrm{~mm} \times 1 \mathrm{~mm})$ was created by keeping the eggs horizontally. This window is to access the CAM tissue and loading the test and standard drug. A methylcellulose disk loaded either with the normal saline, standard or the various concentrations of test drug was kept on top of the CAM tissue (yellowish forge wheel like structure) membrane under the sterile condition. Subsequently, eggs were sealed with the cellophane tape. On the $14^{\text {th }}$ day of incubation, the eggs were taken out from the incubator and the CAM tissues directly beneath each filter paper was resected with the help of forceps and pictures were captured under the microscope.

Total three pictures were captured for each egg i.e. after creating a square window, after placing methylcellulose disk and on the day $14^{\text {th }}$ after removal of the disk. All the procedures were carried out in sterile conditions. A total of 18 eggs were used for each dose for the selected test samples. (Figure 1)

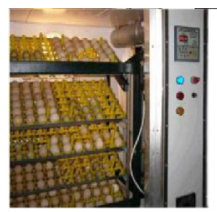

a)

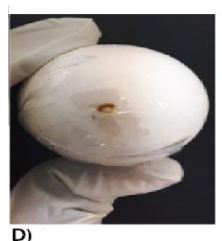

D)

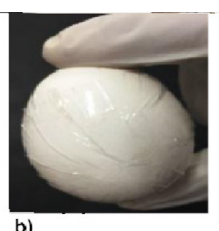

b)

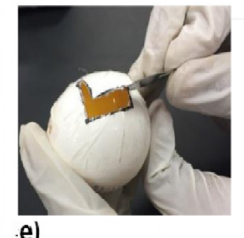

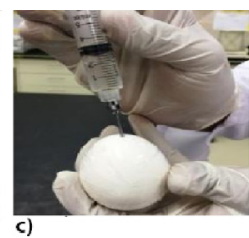

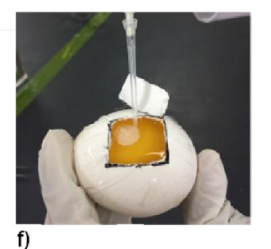

Figure 1: Represents the CAM assay procedure- a) Incubation of eggs for 3 days; b) covering the eggs with cellophane tape; c) removal of albumin from the narrow end of the egg ( $3^{\text {rd }}$ Day); $d$ ) resealing of the drilled hole; e) creation of window on the eggs ( $7^{\text {th }}$ Day); f) placement of methylcellulose disk and drug delivery ( $7^{\text {th }}$ Day).

\section{Drug delivery}

Methylcellulose disk was used to deliver the sample to the CAM tissue. Methylcellulose disk was prepared by methylcellulose solution by placing in the hollow mold of $5 \mathrm{~mm}$ diameter and dried at $37^{\circ} \mathrm{C}$ for $1 \mathrm{hr}$ under vacuum hood. The dried disks were taken out from the molds and $10 \mu \mathrm{l}$ volume of each (which contain the desired quantity of the test and standard drugs) samples were applied on the disks then were dried under laminar airflow for next $12 \mathrm{hrs} .{ }^{14}$ The samples were standard Suramin $(50 \mu \mathrm{g}){ }^{15}$ Lisinopril $(0.01,0.1,1 \mathrm{mM}),{ }^{16}$ Ramipril $(0.01,0.1,1 \mathrm{mM}),{ }^{16}$ Losartan $(5,10,50 \mu \mathrm{M})^{17}$ and Valsartan $(40,80,160 \mu \mathrm{M}) .{ }^{18}$ These doses were selected on the basis of previous studies and to evaluate possible differential dose responses. An equivalent volume of normal saline was used as a control. Only one disk was placed on each CAM. (Figure 1)

The anti-angiogenic effect of the test drugs was quantified by comparing the quantity of blood capillaries under the area of the disk. The scale of 0-2 was used for scoring of anti-angiogenic activity.

The average score for each test dose was calculated and the interpretation of anti-angiogenic effect was done as per the earlier literature: ${ }^{19}$

Average score $<0.5=$ no anti-angiogenic effect (inactive).

$0.5 \leq$ average score $\leq 1=$ weak anti-angiogenic effect.

$1<$ average score $<1.5=$ good anti-angiogenic effect.

Average score $\geq 1.5$ = strong anti-angiogenic effect.

The score obtained from above equation was allocated as follows:

$$
\begin{array}{r}
\text { Average score }=\frac{\text { Number of eggs }(\text { score } 2) \times}{\text { Total number of eggs }(\text { score } 0,1,2)}+ \\
\\
\frac{\text { Number of eggs }(\text { score } 1) \times 1}{\text { Total number of eggs }(\text { score } 0,1,2)}
\end{array}
$$

\section{Sponge implantation}

\section{Operating procedure of cannulated discs sponge and inserting}

Polyester-polyurethane sponge discs, 5-mm thick and 1-cm diameter were prepared and used to detect angiogenesis. ${ }^{20}$ One end of the polyvinyl tubing $1.2 \mathrm{~cm}$ long $\times 1.2 \mathrm{~mm}$ internal diameter was pierced in the center of each sponge disc and secured with two 5/0 Ethicon silk sutures so that the capillary tube was at 90 degrees to the disc face. Sponges were soaked $70 \% \mathrm{v} / \mathrm{v}$ ethanol for overnight then sterilized by boiling in distilled water for 15 mins later irradiated with UV light for 20 mins before implantation. Animals were anaesthetized by cocktail of xylazine $(10 \mathrm{mg} / \mathrm{kg})$ and ketamine $(60 \mathrm{mg} / \mathrm{kg})$ via s.c. The dorsal hairs of the animals were initially cut with the seizure and then commercial hair removal cream was applied topically to remove all the hairs at the operative site. The skin cleaned with $70 \%$ ethanol to avoid any infections. A $1.0 \mathrm{~cm}$ long dorsal mid-line incision was made with curved artery forceps and prepared cannulated sponge discs were implanted aseptically into a subcutaneous (s.c.) pouch. The surgery was done on the lateral sides of the vertebral column for implantation of the sponges. A poly-ethylene cannula that was installed inside each sponge disc initially was exteriorized through a needle puncture in the skin and brought into place by Ethicon 5-0 silk suture and then carefully closed with a sterile polyethylene stopper. Postoperatively, animals were closely observed for any kind of suspicious infections and if so, those animals were discarded from the study. All the operated animals were treated with tramadol $(0.9 \mathrm{mg} / \mathrm{kg}, i . m$. $)$ to avoid postoperative pain and distress and were housed in individual cages to avoid any disturbances to the operated side by the other animals. All the test drugs were administered through the installed cannula once in a day for 14 consecutive days excluding the day of operation where no test drug was given. The control group of mice received sterile normal 
saline. Test drugs were administered daily at morning 9.00 am for 14 days to avoid any diurnal variations. (Figure 2)

\section{Vascular Index (Determination of wet weight $(\mathrm{mg})$ and $\mathrm{Hb}$ content of implants)}

The degree of vascularization of the implanted sponge was assessed by determining the hemoglobin content into the sponge, using the Drabkin method. ${ }^{21}$ At the $14^{\text {th }}$ day, 8 hrs later, after the last test dose administration animals were sacrificed by cervical dislocation and the implanted sponges were excised carefully, the cannula was removed from it and wet weight of the sponges were recorded on the sensitive analytical balance. One sponge from each animal (Other sponge was left for histopathological studies) was homogenized in $2 \mathrm{~mL}$ of Drabkin reagent, centrifuged at $12000 \mathrm{rpm}$ for $20 \mathrm{mins}$. The supernatants were filtered through a $0.22 \mathrm{~mm}$ Millipore filter. The spectrophotometer was used to determine the $\mathrm{Hb}$ concentration of the samples by calculating absorbance at $540 \mathrm{~nm}$ using an ELISA plate reader. The results were compared against a standard hemoglobin curve. The results were expressed as micrograms of $\mathrm{Hb}$ per milligram wet tissue. ${ }^{22}$

\section{Angiogenic factor (Determination of VEGF)}

The implants were removed on $14^{\text {th }}$ day, 8 hrs after the last dose of the test drug administration. The implants were homogenized in PBS $p \mathrm{H}$ $7.4(2 \mathrm{~mL})$ containing $0.05 \%$ Tween 20 and centrifuged at $10000 \times \mathrm{g}$ for 30 mins. Vascular endothelial growth factor (VEGF) determination was done as per the method prescribed in the previous literatures. ${ }^{20}$ Briefly, the supernatant from each implant were measured in $50 \mu \mathrm{L}$ of the supernatant previously homogenized in Drabkin reagent (to remove hemoglobin) and centrifuged $\left(12,000 \times \mathrm{g}, 20 \mathrm{~min}\right.$ at $\left.4^{\circ} \mathrm{C}\right)$ in that $500 \mu \mathrm{L}$ of PBS $p \mathrm{H} 7.4$ containing $0.05 \%$ Tween 20 was added, centrifuged at $12,000 \times \mathrm{g}$ at $4^{\circ} \mathrm{C}$ for $30 \mathrm{~min}$. The amount of the VEGF in each sample was determined by using Immunoassay Kits and following the manufacturer's protocol. The results are expressed as $\mathrm{pg} / \mathrm{mg}$ wet tissue.

\section{Histological analysis}

Histopathological study was done in the implants. The implants from the saline-treated and test drug-treated mice were carefully removed; mush traction on the implants was avoided to maintain the integrity of the tissue. All the adherent tissue were dissected from it and fixed in formalin (10\% w/v in isotonic saline). Halfway section was done from the mid of the sponge of $6-8 \mu \mathrm{m}$ size and were stained with hematoxylin and eosin (H\&E), processed for light microscopic studies.

\section{Statistical analysis}

Data were expressed as means \pm standard deviation. Statistical analysis was performed using one-way ANOVA followed by Dunnett test. All the results were compared with the control group. Statistical analysis was done by using graph-pad Prism 7 version. The level of significance considered as when $P<0.05$.

\section{RESULTS}

\section{Chick chorioallantoic membrane (CAM) assay Number of branching points}

All the test drugs used in this experiment appeared to nontoxic, inasmuch as all the developing embryos survived till the end of the experiments after placement of drug onto the CAMs. Mortality rate was around $40-60 \%$, observed at the beginning of the experiment. In comparison with the controls, application of the disks impregnated with the standard Suramin $(50 \mu \mathrm{g})$, losartan, $50 \mu \mathrm{M}$ and valsartan $160 \mu \mathrm{M}$ had a remarkable inhibitory effect on angiogenesis. Whereas, ACEIs like ramipril and lisinopril has not shown any observable effect on the vessel growth. (Figure 3)
Losartan at medium $(10 \mu \mathrm{M})$ and high $(50 \mu \mathrm{M})$ dose have shown significant $(P<0.001)$ inhibitory activity where the average numbers of branching points were 16.5 and 6 respectively in comparison to the control group. The other ARB, valsartan also shown significant $(P<0.001)$ attenuation in the number of branching points by average 32.1 and 27.3 at medium and high dose respectively in comparison to the normal control group. The overall effect of Losartan high dose, $50 \mu \mathrm{M}$ was as good as standard Suramin but more than valsartan.

\section{Angiogenic score}

Not both the selected ARBs i.e. Losartan and Valsartan have represented a good angiogenic score. Only Losartan at high dose has shown significant $(P<0.01)$ very good angiogenic score similar to standard Suramin $(P<0.001)$ compared to normal control group. Whereas, valsartan also has shown significant $(P<0.01)$ good angiogenic score but at the high dose compared to the control group. At our experimental conditions, none of the selected ACEIs have not shown any effect on neovascularization. (Figure 4 and 5)

It's important to note here that the scoring system does not considered recently developed micro vessels (After utilization of the medication containing plates on the CAM) and those already present on the seventh day. Hence, $100 \%$ diminution of vascular thickness isn't imaginable by

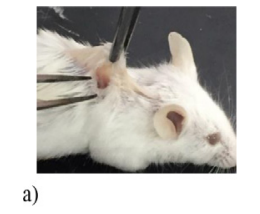

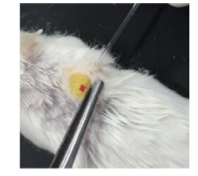

b)

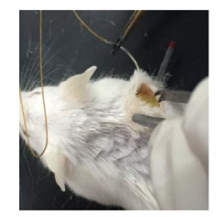

d)

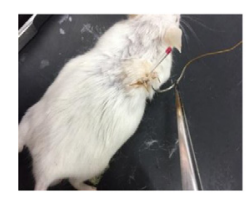

e)

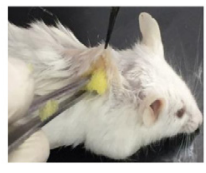

c)
Figure 2: Represents the sponge implantation and cannulation procedure- a) Pouch creation in the dorsal region; b) implantation of sponge; $c$ ) insertion of cannula into the implanted sponge; d) suturing of the skin; e) suturing and positioning of cannula; f)Implantation of cannulated disk on the lateral sides.

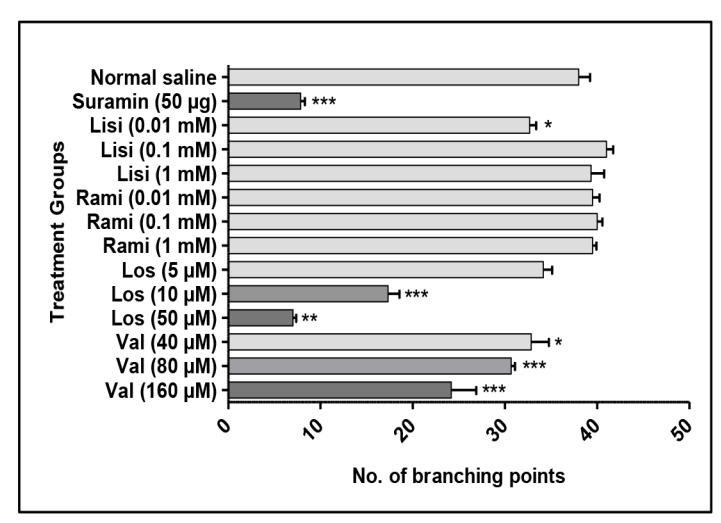

Figure 3: Comparative effect of ACEI and ARBs on the number of branching points of the blood vessels by CAM assay of angiogenesis.

Statistical analysis was performed using one-way ANOVA followed by Dunnett test. All the results were compared with the control group. The level of significance considered as when $\mathrm{P}<0.05$. 
this assay and the number of branching points 16.5 and 6 by the medium and high of losartan was considered as the full attainable limit.

Precisely, no blood capillaries were spotted on the CAM tissue under the disc impregnated with $50 \mu \mathrm{M}$ of losartan, proposing that losartan not only hamper the formation of new micro-vessels but also raze or regression of existing capillaries.

\section{Sponge implantation}

\section{Health assessment during the study}

Health status of the animals was checked periodically to understand any signs of unwanted effects of the test drugs and surgical procedure. Parameters like salivation, lacrimation, diarrhea, body temperature and weight were constant in all the operated animals and there were no signs of the infection at the surgical site were observed.

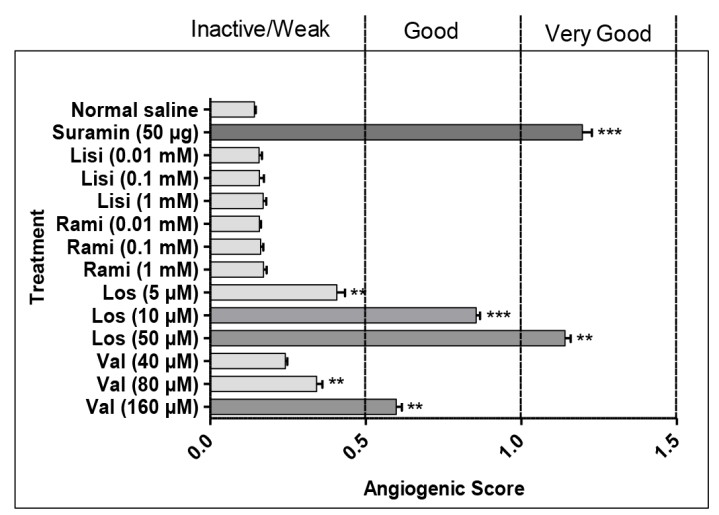

Figure 4: Comparative Anti-angiogenic effect (CAM-assay) of ACEl and ARBs blockers and positive control.

Statistical analysis was performed using one-way ANOVA followed by Dunnett test. All the results were compared with the control group. The level of significance considered as when $P<0.05$.

Pic 1
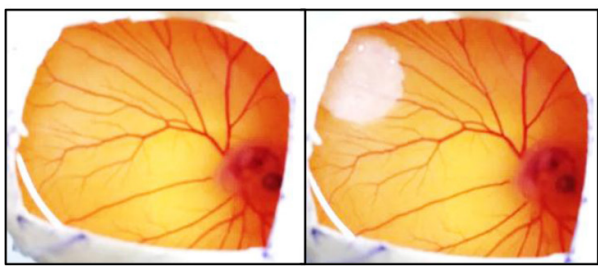

Figure 5.1: Effect of Normal saline on Angiogenesis.

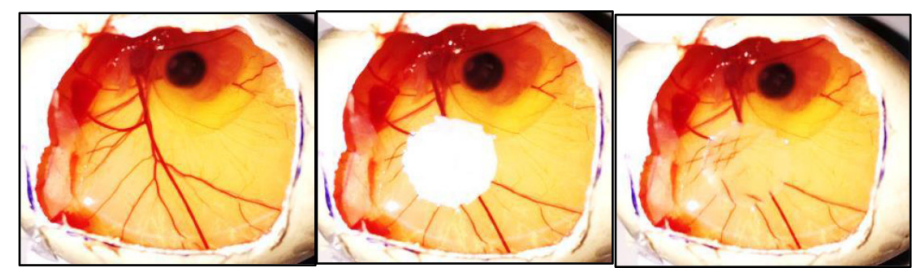

Figure 5.2: Effect of Suramin $50 \mu \mathrm{M}$ on neovascularization by CAM assay.

\section{Wet weight determination of the implants}

During 14 days of the post-operative period, the wet weight of the implants was significantly low in Suramin $(p<0.001)$ treated groups, whereas the wet weight of the implants was increased gradually in the control group. Losartan treated groups were shown significant $(p<0.01)$ gradual decrease in the wet weight in a dose-dependent manner especially at medium $(10 \mu \mathrm{M}, p<0.05)$ and high dose $(50 \mu \mathrm{M}, p<0.01)$ whereas only high dose treated groups of valsartan shown modest decrease in the $\mathrm{Hb}$ level compared to the control groups. No significant changes were observed in ACEI (Lisinopril and Ramipril) treated groups. (Figure 6 and 6.1)

\section{Determination of $\mathrm{Hb}$ Level}

The vascular index was determined by measuring $\mathrm{Hb}$ level in the implants. Fourteen consecutive days administration of test drugs into the sponge implants caused a marked decline in angiogenesis as manifest by decreased in hemoglobin concentration. The decrease in $\mathrm{Hb}$ content was prominent in losartan and standard suramin treated groups, whereas no significant changes were observed in any other groups. There was a modest decline in $\mathrm{Hb}$ content in the valsartan high dose treated group. In the control group, the $\mathrm{Hb}$ level was $2.29 \pm 0.35 \mu \mathrm{gHb} / \mathrm{mg}$ wet tissue $(n=6)$; whereas in the losartan-treated group the level were $2.53 \pm 0.26,2.21 \pm 0.046$, $1.27 \pm 0.109 \mu \mathrm{gHb} / \mathrm{mg}$ in the low, medium and high dose respectively. No other ACEI and ARB have shown any significant change in the Hb level in the implants. (Figure 7)

\section{Effect of VEGF levels}

Vascular endothelial growth factor (VEGF) is considered a key regulator in neovascularization, level of this cytokine has great influence on the microvessel formation, so measurement of VEGF was considered as worthful in this research. VEGF is an important angiogenic factor ${ }^{23}$ and is considered as the main stimulatory factor in tumor angiogenesis and overall neovascularization. Our findings reveals that VEGF level significantly $(P<0.001)$ declined in the standard suramin treated group and in losartan-treated groups at medium $(10 \mu \mathrm{M})$ and high $(50 \mu \mathrm{M})$ doses in a dose-dependent manner compared to normal saline treated group. Valsartan at high dose $(160 \mu \mathrm{M})$ also shown an only modest reduction in the VEGF level compared to the normal group. (Figure 8)

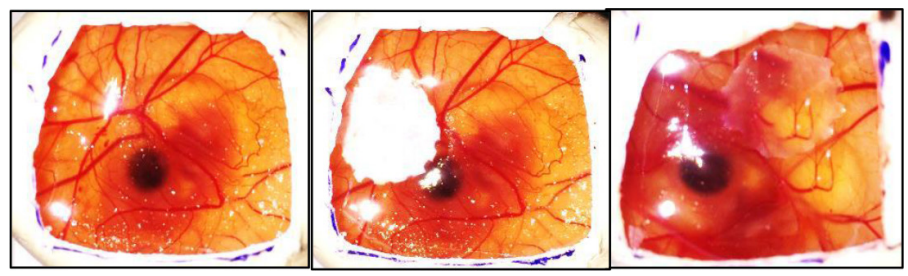

Figure 5.3: Effect of Losartan $5 \mu \mathrm{M}$ on neovascularization by CAM assay.

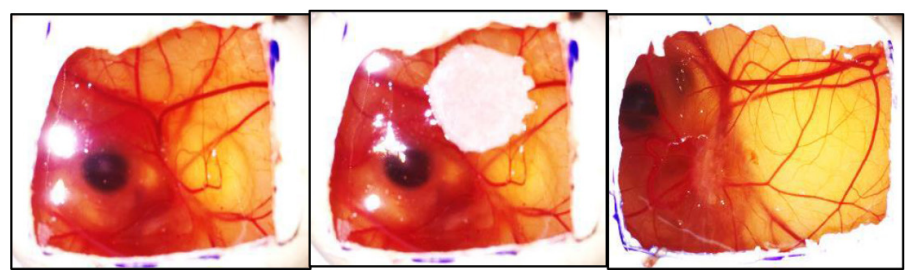

Figure 5.4: Effect of Losartan $10 \mu \mathrm{M}$ on neovascularization by CAM assay. 


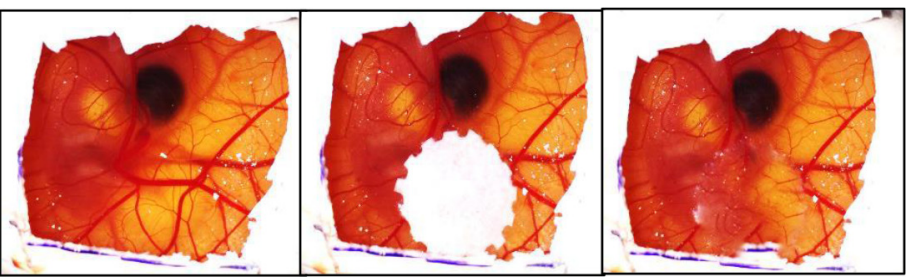

Figure 5.5: Effect of Losartan $50 \mu \mathrm{M}$ on neovascularization by CAM assay.

Figure 5: Picture 1 was captured on $7^{\text {th }}$ day immediately after creating window; picture 2 was captured on the $7^{\text {th }}$ day after loading the sample on the methylcellulose disk and picture 3 was captured after on $14^{\text {th }}$ day of study.

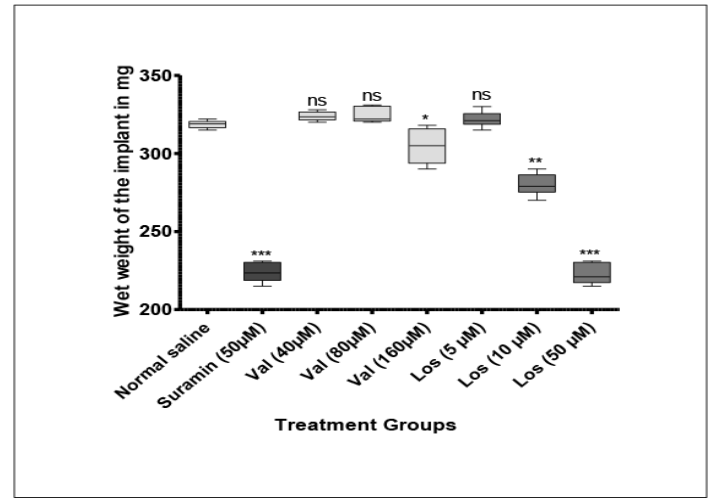

Figure 6: Wet weight of the implants.

Data were expressed as means \pm standard deviation. Statistical analysis was performed using one-way ANOVA followed by Dunnett test. All the results were compared with the control group. Statistical analysis was done by using graph-pad Prism 7 version. The level of significance considered as when $P<0.05$. a

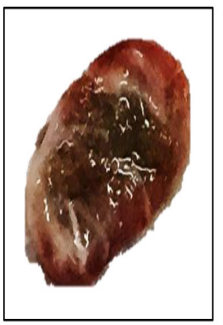

b

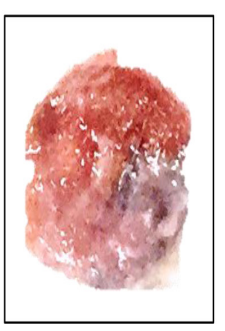

c

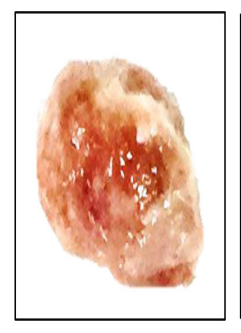

d

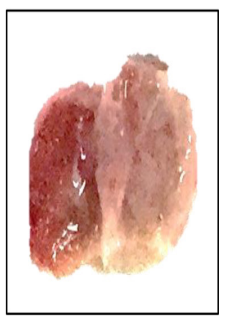

Figure 6.1: Represents the extroverted sponges from the mice.

a) Normal saline treated

b) Suramin treated

c) Losartan high dose $(50 \mu \mathrm{M})$ treated

d) Valsartan high dose $(160 \mu \mathrm{M})$.

\section{Histopathological study}

Sectioning study was carried out for additional confirmation of the above findings in the implants, representing that number of microcapillaries are significantly lower in standard suramin treated group and the high dose of losartan-treated groups compared to saline-treated group. No significant changes were observed in any of the test drugs with respect to the number of microvascular densities. (Figure 9)

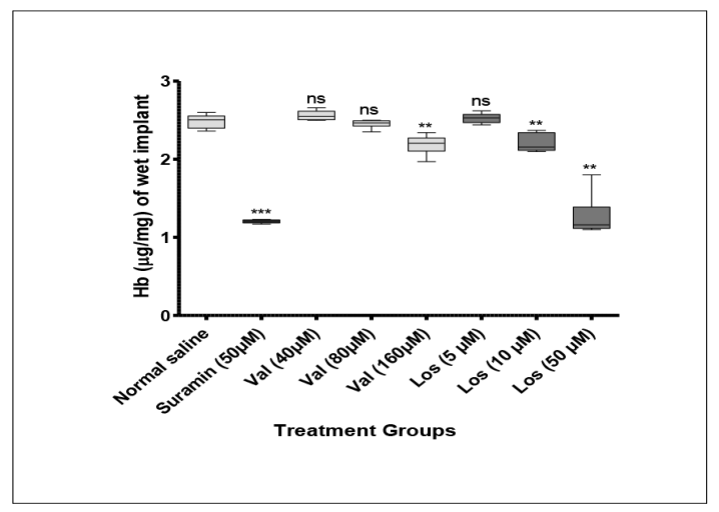

Figure 7: Hemoglobin $(\mathrm{Hb})$ content in implants.

Data were expressed as means \pm standard deviation. Statistical analysis was performed using one-way ANOVA followed by Dunnett test. All the results were compared with the control group. Statistical analysis was done by using graph-pad Prism 7 version. The level of significance considered as when $P<0.05$.

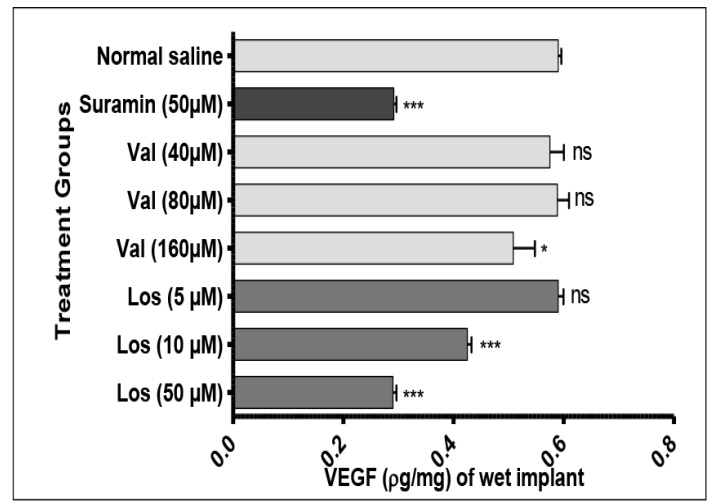

Figure 8: VEGF content in the implant.

Data were expressed as means \pm standard deviation. Statistical analysis was performed using one-way ANOVA followed by Dunnett test. All the results were compared with the control group. Statistical analysis was done by using graph-pad Prism 7 version. The level of significance considered as when $P<0.05$.
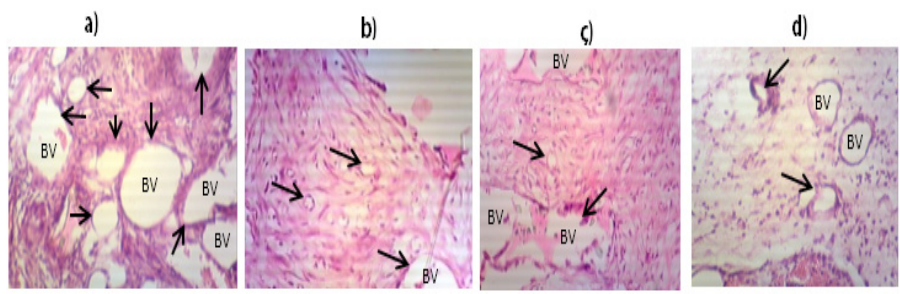

a) Normal saline b) Suramin c) Valsartan high dose d) Losartan high dose Figure 9: Histopathological study of implants- Normal saline treated group represents the crowded sponge matrix; inflammatory cells, fibroblasts and blood vessels compared to the suramin and at high dose of losartan treated groups. BV: Blood vessel.

\section{DISCUSSION}

Epidemiologic and biochemical evidence suggests that is a close association between angiotensin II, angiogenesis, tumor formation and role of ACEIs and angiotensin-receptor blockers (ARBs)., ${ }^{2,3}$ Both the 
categories of the selected drugs show their effect by modulating RAAS, finally attenuating the pathological effects of angiotensin II. So, a possibility arises ACEI and ARBs may possess anti-angiogenic property and our hypothesis also supported by the clinical finding stating the role of ARBs and ACEs in cancer. ${ }^{8-11}$

A case-control study reported that use of ACEIs declines the risk of developing solid tumors, including esophageal, pancreatic and colorectal cancers and reduced risks of basal or squamous cell carcinoma. ${ }^{24}$ Furthermore, patients suffering from advanced stage of non-small-cell lung cancer stated that use of ACEIs in the therapy may prolong the survival. ${ }^{25}$ Surprisingly, a recently conducted population based study showed the use of ACEIs is associated with an increased risk of lung cancer. ${ }^{26}$ However, few researchers have reported that there is no association between the use of ACEIs and ARBs in the risk of cancer. ${ }^{27-28}$ By considering the above facts together we have designed this study to pinpoint the role of these drugs at the cellular level of neovascularization associated with solid tumors. To the best of our information, this is the first study using the CAM model and sponge implantation model to investigate the anti-angiogenic properties of ACEIs (Ramipril, Lisinopril) and ARBs (Losartan, Valsartan). We have screened both the categories of the drugs at three different dose levels by using in-ovo (CAM assay) and in-vivo (sponge implantation technique) models.

We found that none of the ACEIs has shown any anti-angiogenic effect against both the models of angiogenesis. Losartan has shown very good antiangiogenic activity in both the models as good as standard suramin and better then valsartan. Valsartan also mitigates angiogenic response but not as good as standard suramin and losartan.

Our study represents that losartan had shown significant $(p<0.001)$ decline in the number of microvessel formation, number of branching points and significant $(p<0.001)$ increase in angiogenic score in all three doses in a dose-dependent manner compared to the control group.

In the developing CAM the angiogenic growth occurs within the mesenchyme and test drugs are placed on the existing microvessel, therefore angiogenesis cannot be distinguished from increased vascular density. ${ }^{29}$ Moreover, the test drugs were loaded through the methylcellulose disk so nonspecific inflammatory reactions may develop which induce a secondary angiogenic response. ${ }^{30}$ In spite of these drawbacks, the CAM assay model from long back has been preferred in-vivo method for angiogenic studies.

In CAM assay hypoxia, ischemia and free radical formation are provoking factors in the collateral growth development and angiogenesis. During the ischemic phase various growth factors and proteinases are produced causes vascular smooth muscle cells to migrate and divide especially Hypoxia-inducible transcription factors (HIF) and VEGF play critical roles in collateral growth and angiogenesis. ${ }^{31}$

The protective effect offered by ARBs may be because of its free radical scavenging property. Earlier studies suggested that losartan is sufficiently lipophilic in nature so that it may freely penetrate cell membranes. ${ }^{32} \mathrm{On}$ the other hand, valsartan appears to bind tightly to AT1 but remains at the cell surface..$^{33}$ Because of this pharmacokinetic variation losartan has offered better effect than valsartan.

As stated above ischemia causes the release of VEGF and HIF which induces angiogenesis so measurement of VEFG was consider as one of the crucial parameters in this study. VEGF was found significantly low in losartan treated group in sponge implantation model of angiogenesis. In angiogenesis, proliferation and migration of vascular endothelial cells are important steps and are decisively controlled by many extracellular stimuli. Proangiogenic intracellular signaling cascades involve many messengers and generate a rise in intracellular calcium ions. In the cell physiology, $\mathrm{Ca}^{2+}$ plays many important roles. The extra and intracellular concentration of $\mathrm{Ca}^{2+}$ ions determines many cell functions thus slight variation may change in the cell response so their concentration is controlled precisely. Intracellular $\mathrm{Ca}^{2+}$ is one of a vital second messenger in the process of cell division and the slight change in concentration activate the transduction of growth-related signals by activating many growth factor proteins. A rise in intracellular $\mathrm{Ca}^{2+}$ ions by two mechanisms viz entry from extracellular medium, through the opening of calcium-permeable channels in the plasma membrane or release from intracellular $\mathrm{Ca}^{2+}$ stores, which dependent on store-operated $\mathrm{Ca}^{2+}$ channels (SOCC) or non-store-operated $\mathrm{Ca}^{2+}$ channels (NSOCC). ${ }^{34}$ Previous literatures reveal that losartan inhibits the $\mathrm{Ca}^{2+}$ entry induced by Ag-II by blocking NSOCC and thus hampering angiogenesis. ${ }^{35}$

None of the ACEIs has shown any effect with respect to the wet weight of the sponge, $\mathrm{Hb}$ and VEGF content in the implants. Losartan at all the three doses $(5,10$ and $50 \mu \mathrm{M})$ has shown a dose-dependent decline in the vascular index and angiogenic factor similar to standard suramin $(50 \mu \mathrm{M})$ but better the valsartan. However, valsartan at $160 \mu \mathrm{M}$ concentration was a poor inhibitor of angiogenesis. This variation in selected ARBs may be because of their differences in pharmacokinetics, antioxidant potency and inhibitory effect on VEGF level.

\section{CONCLUSION}

Based upon our finding we conclude that ARBs i.e. losartan and valsartan both possess anti-angiogenic activity and both the ACEIs i.e. lisinopril and ramipril has not shown any effect on the neovascularization. Among the ARBs, losartan has shown a potent anti-angiogenic effect in a dosedependent manner against both the models while valsartan has shown a modest anti-angiogenic effect that to at high dose.

From decades, losartan and valsartan are already in the clinical practice for many complications and safety profiles of these drugs are already defined. Our new findings propose further new directions for the use of these drugs in the angiogenesis research.

\section{ACKNOWLEDGEMENT}

The author gratefully acknowledges the approval and the support of this research study by grant no. 7067-PHM-2017-1-7-F from the Deanship of Scientific Research at Northern Border University, Arar. K.S.A.

\section{CONFLICT OF INTEREST}

The authors declare no conflict of interest.

\section{ABBREVIATIONS}

ACE: Angiotensin Converting Enzyme; ACEI: Angiotensin Converting Enzyme Inhibitors; ARB: Angiotensin Receptor Blockers; AT1R: Angiotensin 1 Receptor; BV: Blood Vessel; CAM: Chick Embryo Chorioallantoic Membrane; H\&E: hematoxylin and eosin; Hb: Hemoglobin; HIF: Hypoxia-Inducible Transcription Factors; i.m.: Intramascular; LCEB: Local Committee of Bio Ethics; Lisi: Lisinopril; Los: Losartan; mg: Milligram; mM: Millimolar; NCEB: National Committee of Bio Ethics; NSOCC: non-store-operated Ca2+ channels; PBS: phosphate buffered solution ; Pg: Picogram; RAAS: Renin-Angiotensin-Aldosterone-System; Rami: Ramipril; s.c.: Subcutaneous; SOCC: Store-Operated Ca2+ Channels; Val: Valsartan; VEGF: Vascular Endothelial Growth Factor; $\mu \mathrm{g}$ : Microgram; $\mu \mathrm{M}$ : Micromolar.

\section{REFERENCES}

1. Folkman J, Cotran R. Relation of vascular proliferation of tumour growth. Int Rev Exp Pathol. 1976;16:207-48 
2. Tahergorabi Z, Khazaei M. A review on angiogenesis and its assays. Iran J Basic Med Sci. 2012;15(6):1110-26.

3. George AJ, Thomas WG, Hannan RD. The renin-angiotensin system and cancer: old dog, new tricks. Nat Rev Cancer. 2010;10(11):745-59.

4. Rosenthal T, Gavras I. Angiotensin inhibition and malignancies: a review. J Hum Hypertens. 2009;23(10):623-35.

5. Tamarat R, Silvestre JS, Durie M, Levy BI. Angiotensin II angiogenic effect in vivo involves vascular endothelial growth factor-and inflammation-related pathways. Lab Invest. 2002;82(6):747-56.

6. Suganuma T, Ino K, Shibata K, Kajiyama H, Nagasaka T, Mizutani S, et al. Functional expression of the angiotensin II type 1 receptor in human ovarian carcinoma cells and its blockade therapy resulting in suppression of tumor invasion, angiogenesis and peritoneal dissemination. Clin Cancer Res. 2005; $11(7): 2686-94$

7. Fujita M, Hayashi I, Yamashina S, Fukamizu A, Itoman M, Majima M. Angiotensin type 1a receptor signaling-dependent induction of vascular endothelial growth factor in stroma is relevant to tumor-associated angiogenesis and tumor growth. Carcinogenesis. 2005;26(2):271-9.

8. Lever AF, Hole DJ, Gillis CR, McCallum IR, McInnes GT, MacKinnon PL, et al. Do inhibitors of angiotensin-I-converting enzyme protect against risk of cancer? Lancet. 1998;352(9123):179-84.

9. Christian JB, Lapane KL, Hume AL, Eaton CB, Weinstock MA, Trial V. Association of ACE inhibitors and angiotensin receptor blockers with keratinocyte cancer prevention in the randomized VATTC trial. J Natl Cancer Inst. 2008;100(17):1223-32.

10. Jick H, Jick S, Derby LE, Vasilakis C, Myers MW, Meier CR. Calcium-channel blockers and risk of cancer. Lancet. 1997;349(9051):525-8.

11. Sjoberg T, Garcia RLA, Lindblad M. Angiotensin-converting enzyme inhibitors and risk of esophageal and gastric cancer: a nested case-control study. Clin Gastroenterol Hepatol. 2007;5(10):1160-6.

12. Dézsi CA. A review of clinical studies on angiotensin II receptor blockers and risk of cancer. Int J Cardiol. 2014;177(3):748-53.

13. Chidrawar VR, Mohd I. Comparative experimental studies of few L-type and T-type $\mathrm{Ca}^{2+}$ channel blockers against in-ovo and in-vitro models of angiogenesis. Asian Journal of Pharmaceutical Research and Health Care. 2018:10(1):1-9.

14. Dai X, Cui SG, Wang T, Liu Q, Song HJ, Wang R. Endogenous opioid peptides, endomorphin-1 and -2 and deltorphin I, stimulate angiogenesis in the CAM assay. Eur J Pharmacol. 2008;579(1-3):269-7.

15. Danesi R, DelBianchi S, Soldani P, Campagni A, LaRocca RV, Myers CE, et al. Suramin inhibits bFGF-induced endothelial cell proliferation and angiogenesis in the chick chorioallantoic membrane. Br J Cancer. 1993;68(5):932-8.

16. Zakrocka I, Kocki T, Turski WA. The effect of three angiotensin-converting enzyme inhibitors on kynurenic acid production in rat kidney in vitro. Pharmacol Rep. 2017;69(3):536-41.

17. Oh E, Kim JY, Cho Y, An H, Lee N, Jo H, et al. Overexpression of angiotensin II type 1 receptor in breast cancer cells induces epithelial-mesenchymal transition and promotes tumor growth and angiogenesis. Biochim Biophys Acta. 2016;1863(6):1071-81.

18. Wu TT, Niu HS, Chen LJ, Cheng JT, Tong YC. Increase of human prostate cancer cell (DU145) apoptosis by telmisartan through PPAR-delta pathway. Eur J Phar- macol. 2016;775:35-42.

19. Bürgermeister J, Paper DH, Vogl H, Linhardt RJ, Franz G. LaPSvS1, a (1->3)beta-galactan sulfate and its effect on angiogenesis in vivo and in vitro. Carbohydr Res. 2002;337(16):1459-66

20. Saraswati S, Kanuajia PK, Kumar S, et al. Tylophorine, a phenanthraindolizidine alkaloid isolated from Tylophora indica exerts antiangiogenic and antitumo activity by targeting vascular endothelial growth factor receptor 2-mediatedangiogenesis. Mol Cancer. 2013;12(1):82.

21. Lage AP, Andrade SP. Assessment of angiogenesis and tumor growth in con-scious mice by a fluorimetric method. Microvasc Res. 2000;59(2):278-85.

22. Stewart GM, PeterWH. Angiogenesis Protocols. Methods in Molecular Biology 1430. The Sponge Implant Model of Angiogenesis. Springer Science Business Media New York, Third Edition, Humana press. 2016;333-43.

23. Yancopoulos GD, Davis S, Gale NW, Rudge JS, Wiegand SJ, Holash J. Vascularspecific growth factors and blood vessel formation. Nature. 2000;407(6801):242-8

24. Lang L. ACE inhibitors may reduce esophageal cancer incidence. Gastroenterology 2006;131(2):343-4.

25. Wilop S, Hobe SV, Crysandt M, Esser A, Osieka R, Jost E. Impact of angiotensin I converting enzyme inhibitors and angiotensin II type 1 receptor blockers on survival in patients with advanced non-small-cell lung cancer undergoing $1^{\text {st }}$-line platinum-based chemotherapy. J Cancer Res Clin Oncol. 2009;135(10):1429-35.

26. Hicks BM, Filion KB, Yin H, Sakr L, Udell JA, Azoulay L. Angiotensin converting enzyme inhibitors and risk of lung cancer: population-based cohort study. BMJ. 2018;24:363.

27. Coleman C, Baker W, Kluger J, White CM. Antihypertensive medication and their impact on cancer incidence: a mixed treatment comparison meta-analysis of randomized controlled trials. J Hypertens. 2008;26(4):622-9.

28. Koomen E, Herings R, Guchelaar H, Nijsten T. Melanoma incidence and exposure to angiotensin-converting enzyme inhibitors and angiotensin receptor blockers. Cancer Epidemiol. 2009;33(5):391-5.

29. Knighton DR, Fiegel VD, Phillips GD. The assay of angiogenesis. Prog Clin Biol Res. 1991;365:291-9.

30. Splawinski J, Michna M, Palczak R, Konturek S, Splawinska B. Angiogenesis: quantitative assessment by the chick chorioallantoic membrane assay. Methods Find Exp Clin Pharmacol. 1988;10(4):221-6.

31. Heil M, Ziegelhoeffer T, Pipp F, Kostin S, Martin S, Clauss M, et al. Blood monocyte concentration is critical for enhancement of collateral artery growth. Am J Physiol Heart Circ Physiol. 2002;283(6):H2411-9.

32. Peters J, Obermüller N, Woyth A, Peters B, Maser-Gluth C, Kränzlin B, et al. Losartan and angiotensin II inhibit aldosterone production in anephric rats via different actions on the intraadrenal renin-angiotensin system. Endocrinology. $1999 ; 140(2): 675-82$

33. Fierensa FLP, Vanderheyden PML, Roggeman C, DeBacker J, Thekkumkara TJ Vauquelin G. Tight binding of the angiotensin AT1 receptor antagonist. Biochem Pharmacol. 2001;61(10):1227-35.

34. Rahman AU, Zaman K, editors. Topics in Anti-Cancer Research. Bentham Science Publishers. 2014.

35. Guo RW, Yang LX, Li MO, Pan XH, Liu B, Deng YL. Stim1- and Orai1-mediated store-operated calcium entry is critical for angiotensin II-induced vascular smooth muscle cell proliferation. Cardiovasc Res. 2012;93(2):360-70

Article History: Submission Date : 20-11-2018; Revised Date : 03-01-2019; Acceptance Date : 25-01-2019

Cite this article: Chidrawar VR, Soomro S. Comparative Experimental Studies of ACEls and ARBs against Two Different Models of Angiogenesis. J Young Pharm. 2019;11(2):165-71. 